\title{
A Hybrid Control Scheme for Adaptive Live Streaming
}

\author{
Huan Peng \\ Communication University of China \\ hpeng@cuc.edu.cn \\ Yongbei Yang \\ Communication University of China \\ vrwhatvdo@cuc.edu.cn
}

\author{
Yuan Zhang \\ Communication University of China \\ yzhang@cuc.edu.cn \\ Jinyao Yan \\ Communication University of China \\ jyan@cuc.edu.cn
}

\begin{abstract}
The live streaming is more challenging than on-demand streaming, because the low latency is also a strong requirement in addition to the trade-off between video quality and jitters in playback. To balance several inherently conflicting performance metrics and improve the overall quality of experience (QoE), many adaptation schemes have been proposed. Bitrate adaptation is one of the major solutions for video streaming under time-varying network conditions, which works even better combining with some latency control methods, such as adaptive playback rate control and frame dropping. However, it still remains a challenging problem to design an algorithm to combine these adaptation schemes together. To tackle this problem, we propose a hybrid control scheme for adaptive live streaming, namely HYSA, based on heuristic playback rate control, latency-constrained bitrate control and QoE-oriented adaptive frame dropping. The proposed scheme utilizes KaufmanâĂŹs Adaptive Moving Average (KAMA) to predict segment bitrates for better rate decisions. Extensive simulations demonstrate that HYSA outperforms most of the existing adaptation schemes on overall QoE.
\end{abstract}

\section{CCS CONCEPTS}

-Information systems $\rightarrow$ Multimedia streaming; Information systems applications; Multimedia information systems.

\section{KEYWORDS}

live streaming; bitrate adaptation; playback rate control; frame dropping

\section{ACM Reference Format:}

Huan Peng, Yuan Zhang, Yongbei Yang, and Jinyao Yan. 2019. A Hybrid Control Scheme for Adaptive Live Streaming. In Proceedings of the 27th ACM International Conference on Multimedia (MM '19), October 21-25, 2019, Nice, France. ACM, New York, NY, USA, 5 pages. https://doi.org/10.1145/ 3343031.3356049

Permission to make digital or hard copies of all or part of this work for personal or classroom use is granted without fee provided that copies are not made or distributed for profit or commercial advantage and that copies bear this notice and the full citation on the first page. Copyrights for components of this work owned by others than ACM must be honored. Abstracting with credit is permitted. To copy otherwise, or republish, to post on servers or to redistribute to lists, requires prior specific permission and/or a fee. Request permissions from permissions@acm.org.

MM '19, October 21-25, 2019, Nice, France

(C) 2019 Association for Computing Machinery.

ACM ISBN 978-1-4503-6889-6/19/10 . \$ \$15.00

https://doi.org/10.1145/3343031.3356049

\section{INTRODUCTION}

Recent years have seen tremendous growth of live streaming applications. Different from on-demand streaming, live streaming has tight latency constraints. It's very challenging to reduce the latency while maintaining high video quality and smooth playback. Bitrate adaptation is the most common solution for improving the QoE of video streaming under time-varying network conditions. However, the existing adaptive bitrate algorithms, such as BOLA [6], RobustMPC [8], Pensieve [3] and Oboe [1], haven't taken the latency into account. In addition to adapting the video bitrate, playback rate control and frame dropping are always utilized to reduce the latency in live streaming. Mingfu Li et al. [2] employed playback rate adaptation, and Miller et al. [4] and Shen Y et al. [5] adopted frame dropping to reduce the latency. However, these methods lack the capability to balance between latency and video quality, without fully considering all aspects of QoE.

In this paper, we propose HYSA, an effective hybrid control scheme to realize playback rate adaptation, bitrate adaptation and frame dropping adaptation. First, the playback rate is adaptively adjusted with a buffer-based heuristic method. Then, taking advantage of the playback rate decisions and KAMA-based predicted segment bitrates, we propose the latency-constrained bitrate adaptation scheme to make optimal bitrate decisions for the QoE-oriented frame dropping adaptation scheme in the next step. Our extensive simulation results demonstrate that the proposed HYSA outperforms existing adaptation schemes on the overall QoE.

The rest of the paper is organized as follows. Section 2 introduces the system framework in live streaming scenario and the simulation platform. Section 3 describes the details of the proposed hybrid control scheme. In Section 4, the performance of our method is evaluated comprehensively. Section 5 concludes this paper.

\section{SYSTEM OVERVIEW}

Figure 1 depicts a typical scenario of live streaming. The video frames generated in real-time are uploaded to a transcoding server, which re-encodes the video into multiple representations, each at a different bitrate. These representations are then transmitted to CDN (Content Delivery Network) nodes, which act as edge servers. The client decides which representation to download from one of the CDN nodes based on some state information, such as buffer occupancy and throughput. Besides, the client can adaptively adjust its playback rate and skip some frames to reduce the latency.

The simulator simulates the downloading of video frames under various network conditions and the adaptive playback of a player. The simulator takes video trace, network trace and decisions from 


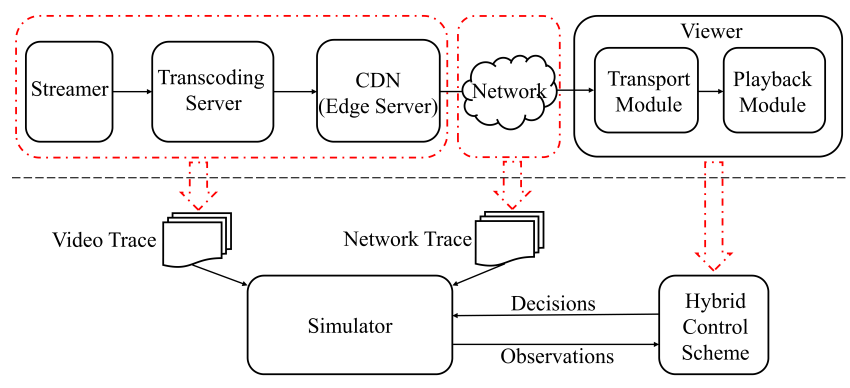

Figure 1: Architecture of live streaming system and the simulator

the hybrid control scheme as inputs. The video trace records the size of video frames and their time of arrival at $\mathrm{CDN}$, while the network trace simulates throughputs of the downloading network. The simulator collects observations after downloading every frame, then the control scheme makes decisions after downloading a complete group of pictures (GOP) by taking advantage of these observations. Hereinafter we use a segment to refer to a GOP.

\section{HYBRID CONTROL SCHEME}

In this section, we describe the details of the proposed hybrid control scheme - HYSA, which consists of segment bitrate prediction module, playback rate control module, bitrate control module and frame dropping control module, as shown in Figure 2.

\subsection{QoE model}

Many studies have highlighted the critical role that QoE plays in the design of adaptation schemes. Here we refer to the QoE model specified by the grand challenge in ACM MM 2019 ${ }^{1}$, which mainly focuses on five performance metrics: video quality, rebuffering, latency, frame skipping and quality switching. Their impacts on QoE are notated by QoE quality, $Q o E_{\text {rebuf }}, Q o E_{\text {latency, }}, Q o E_{\text {skip }}$ and $Q o E_{\text {switch }}$ respectively. The overall QoE is calculated as follows:

$$
\begin{aligned}
Q o E= & Q o E_{\text {quality }}+Q o E_{\text {rebuf }}+Q o E_{\text {latency }}+Q o E_{\text {skip }} \\
& +Q o E_{\text {switch }} \\
= & \sum_{k=1}^{K}\left(p_{q} V_{k} d_{f}-p_{r} t_{k}^{r}-p_{l} l_{k}-p_{s} t_{k}^{s}-p_{w}\left|V_{k}-V_{k-1}\right|\right)
\end{aligned}
$$

where $K$ is the total number of frames. The coding bitrate of frame $k$ is notated by $V_{k}$, while $d_{f}$ is its length. $t_{k}^{r}$ and $t_{k}^{s}$ denote the rebuffering duration and video length skipped when downloading frame $k$ respectively, and $l_{k}$ is the latency. $p_{q}, p_{r}, p_{l}, p_{s}, p_{w}$ are weight factors used to describe the importance of corresponding QoE metrics. $\left|V_{k}-V_{k-1}\right|$ describes the quality variation of two adjacent video frames.

\subsection{Segment Bitrate Prediction}

The next download duration can be estimated if the next segmentâĂŹs actual bitrate is known. However, it isnâÁŹt realistic to get information about the video that hasnâĂŹt been generated in live streaming. Therefore, most of the existing algorithms estimate the

\footnotetext{
${ }^{1}$ https://www.aitrans.online/MMGC/
}

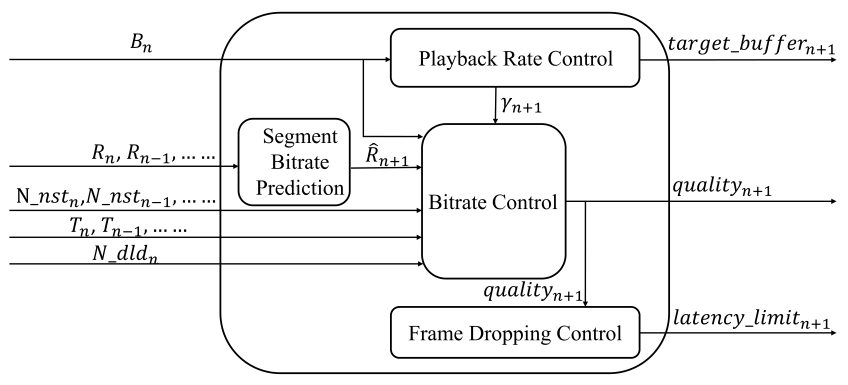

Figure 2: Overview of the proposed hybrid control scheme

next download duration using the coding bitrate of the upcoming segment instead of its actual bitrate, ignoring the fact that segment's actual bitrate varies significantly for a given coding bitrate, as indicated by Figure 3. Besides, we can observe that for segments in two videos with the same content but different qualities, the ratios of their actual bitrates have a similar trend with the ratios of their coding bitrates.

Assume that each segment is edcoded at $M$ different bitrates, and let $V_{n, m}$ and $R_{n, m}$ be the coding bitrate and the actual bitrate of segment $n$ at quality level $m$, satisfying $V_{n, m_{1}}<V_{n, m_{2}}, R_{n, m_{1}}<$ $R_{n, m_{2}}, \forall m_{1}<m_{2}$. For the $n$-th segment already downloaded at quality $q_{n}$, we can estimate its actual bitrates of other quality levels based on the above observation:

$$
R_{n, m} \approx \frac{V_{n, m}}{V_{n, q_{n}}} R_{n, q_{n}}, m \neq q_{n} \text { and } m \in[1, M]
$$

Here we employ KaufmanâĂŹs Adaptive Moving Average (KAMA) to predict the actual bitrates of the upcoming segment. The bitrate of the next segment $n+1$ at quality level $m$, denoted as $\hat{R}_{n+1, m}$, can be predicted as follows:

$$
\hat{R}_{n+1, m}=\left(1-S C_{n}\right) \hat{R}_{n, m}+S C_{n} R_{n, m}
$$

The smoothing factor $S C_{n}$ is dynamically calculated for every sample, i.e. segment bitrate. To get the smoothing factor, we first set two boundaries for it, based on the method of calculating smoothing factor in Exponential Moving Average (EMA):

$$
\begin{aligned}
& S C_{\text {slowest }}=\frac{2}{l_{\max }+1} \\
& S C_{\text {fastest }}=\frac{2}{l_{\min }+1}
\end{aligned}
$$

The $l_{\max }$ and $l_{\min }$ are the number of samples for the slowest and fastest EMA respectively. Then we calculate the efficiency ratio $E R_{n}$, which shows the efficiency of sample fluctuations.

$$
E R_{n}=\frac{\left|R_{n, m}-R_{n-N_{1}, m}\right|}{\sum_{i=0}^{N_{1}-1}\left|R_{n-i, m}-R_{n-i-1, m}\right|}
$$

where $N_{1}$ specifies the number of samples used for calculating $E R_{n}$, and $E R_{n}$ is always between 0 and 1 . Using $E R_{n}$ and two boundaries, the $S C_{n}$ can be derived as below:

$$
S C_{n}=\left[E R_{n}\left(S C_{\text {fastest }}-S C_{\text {slowest }}\right)+S C_{\text {slowest }}\right]^{2}
$$




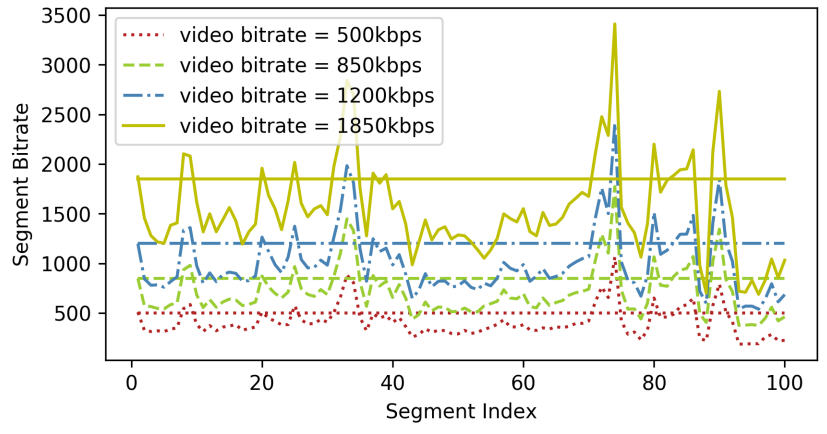

Figure 3: Actual bitrates of segments at variable coding bitrates

\subsection{Playback Rate Control}

In the playback rate control module, the target buffer is introduced to help control playback rate $\gamma$. The so-called target buffer is marked as $\left[B_{\min }, B_{\text {target }}, B_{\max }\right]$, where $B_{\min }$ and $B_{\max }$ form a buffer interval that player can play buffered video at normal playback rate, and $B_{\text {target }}$ means the target buffer occupancy to resume playback when interruptions occur. When the buffer occupancy is below $B_{\min }$, the player will slow down the playback rate to 0.95 , while speeding up the playback rate to 1.05 when the buffer occupancy is above $B_{\max }$. In the grand challenge, the target buffer can only be set to 0 or 1, i.e. $\left[B_{\text {min }}^{0}, B_{\text {target }}^{0}, B_{\text {max }}^{0}\right]$ or $\left[B_{\text {min }}^{1}, B_{\text {target }}^{1}, B_{\text {max }}^{1}\right]$, satisfying $B_{\text {min }}^{0}<B_{\text {min }}^{1}<B_{\text {target }}^{0}<B_{\text {target }}^{1}<B_{\text {max }}^{0}<B_{\text {max }}^{1}$.

The heuristic playback rate control module decides which target buffer to choose solely depending on current buffer occupancy $B_{n}$. Five cases are considered, as described below:

Case $1: B_{n}<B_{\text {min }}^{0}$, which means there is a substantial risk of interruptions, and $\gamma=0.95$ whichever target buffer is chosen. To restart playback as soon as possible, the target buffer is set to 0 due to smaller $B_{\text {target }}$.

Case $2: B_{n} \in\left[B_{\text {min }}^{0}, B_{\text {min }}^{1}\right)$, which means stalls may be encountered even though it is less possible than Case 1. Therefore, the target buffer is set to 1 to make player slow down the playback rate to 0.95 .

Case 3: $B_{n} \in\left[B_{\text {min }}^{1}, B_{\text {max }}^{0}\right)$, which means buffer occupancy remains in a reasonable interval, and $\gamma=1.0$ whichever target buffer is chosen.

Case $4: B_{n} \in\left[B_{\text {max }}^{0}, B_{\text {max }}^{1}\right)$, which means the latency is relatively large due to buffered video. Therefore, the target buffer is set to 0 to speed up the playback rate to 1.05 .

Case 5: $B_{n} \geq B_{\text {max }}^{1}$, which means large latency caused by buffered video, and $\gamma$ is equal to 1.05 whichever target buffer is chosen.

Based on the discussions above, the playback rate can be adjusted by the target buffer as follows:

$$
\begin{gathered}
\text { target_buffer }_{n+1}= \begin{cases}1, & \text { if } B_{n} \in\left[B_{\text {min }}^{0}, B_{\text {max }}^{0}\right) \\
0, & \text { otherwise }\end{cases} \\
\gamma_{n+1}= \begin{cases}0.95, & \text { if } B_{n} \in\left[0, B_{\text {min }}^{1}\right) \\
1.0, & \text { if } B_{n} \in\left[B_{\text {min }}^{1}, B_{\text {max }}^{0}\right) \\
1.05, & \text { otherwise }\end{cases}
\end{gathered}
$$

\subsection{Bitrate Control}

The latency-constrained bitrate control module makes bitrate decisions based on some state information, such as buffer occupancy, predicted segment bitrate and playback rate derived from the playback rate control module. The optimal bitrate is selected to minimize $D_{n+1}$, the estimated latency after downloading the next segment.

Denoting segment length by $d$, the duration of downloading the upcoming segment $n+1$ at quality $m$, notated by $T_{n+1}$, can be calculated using the predicted segment bitrate $\hat{R}_{n+1, m}$ and the estimated network throughput $\hat{C}_{n+1}$ derived by Weighted Moving Average as follows:

$$
T_{n+1}=\frac{\hat{R}_{n+1, m} d}{\hat{C}_{n+1}}
$$

During the downloading process, the player will consume buffered video at playback rate $\gamma_{n+1}$ if the buffer isnâÁŹt drained. The buffer occupancy after downloading, namely $B_{n+1}$, can be calculated as:

$$
B_{n+1}=\max \left[B_{n}+d-\gamma_{n+1} T_{n+1}, 0\right]
$$

To estimate the latency caused by video accumulated at CDN after downloading the next segment, we start with estimating the video accumulation speed at CDN, denoted by $\hat{v}_{n+1}$, as follows:

$$
\hat{v}_{n+1}=\beta v_{n}=\beta \frac{N \_n s t_{n}-N \_n s t_{n-1}}{T_{n}} d_{f}
$$

where $\beta$ is a predictive factor, and $N \_n s t_{n}$ indicates the index of the latest frame at CDN after downloading the $n$-th segment. Then, the latency caused by accumulated video at CDN after next downloading interval, namely $D_{-} c d n_{n+1}$, is calculated by:

$$
D \_c d n_{n+1}=\max \left[\left(N \_n s t_{n}-N \_d l d_{n}\right) d_{f}+\hat{v}_{n+1} T_{n+1}-d, 0\right]
$$

where $N \_d l d_{n}$ represents the index of most recently downloaded frame. The objective of the bitrate adaptive algorithm is to find the quality that results in lowest latency without interruptions, which can be described as the following optimization problem:

$$
\begin{gathered}
\text { Minimize } D_{n+1}=B_{n+1}+D_{-} c d n_{n+1} \\
\text { Subject to } B_{n+1}>B_{t h}
\end{gathered}
$$

where $B_{t h}$ is a warning threshold indicating the upcoming stall event. Since there are finite available bitrates, we can get the optimal quality level quality ${ }_{n+1}$ by going through all choices.

\subsection{Frame Dropping Control}

The client can reduce the latency by dropping some frames when current latency is above a specific threshold. We propose a QoEoriented frame dropping method to adaptively adjust the latency threshold that triggers frame skipping. Assuming that the client skips $N$ frames when the latency is above $l_{n+1}$ during next segmentâĂŹs downloading, the positive and negative impact of frame skipping on QoE compared to non-skip, denoted by $Q o E_{p}$ and $Q o E_{n}$, can be estimated based on the given QoE model:

$$
\begin{gathered}
Q o E_{n}=p_{q} V_{n+1, \text { qualit }_{n+1}} d_{f} N+p_{s} d_{f} N \\
Q o E_{p}=p_{d} \lambda l_{n+1} N
\end{gathered}
$$

Here, $\lambda l_{n+1}$ is used to estimate the average latency if frame skipping is not performed when the latency is above $l_{n+1}$. Therefore, when $Q o E_{p}$ is larger than $Q o E_{n}$, frame skipping is a good choice:

$$
p_{d} \lambda l_{n+1} N>p_{q} V_{n+1, \text { quality }_{n+1}} d_{f} N+p_{s} d_{f} N
$$


Table 1: Performance comparison of the adaptation schemes

\begin{tabular}{|c|c|c|c|c|c|c|}
\hline Method & QoE $E_{\text {overall }}$ & QoE $E_{\text {quality }}$ & QoE $E_{\text {rebuf }}$ & QoE $E_{\text {latency }}$ & QoE $E_{\text {skip }}$ & QoE $E_{\text {switch }}$ \\
\hline HYSA & 2424.04 & 3548.00 & -418.92 & -608.32 & -86.28 & -10.44 \\
\hline HYSA-N & 2336.33 & 3398.36 & -398.11 & -571.27 & -82.28 & -10.37 \\
\hline MPC & 2000.44 & 3056.23 & -255.98 & -759.17 & -30.23 & -10.41 \\
\hline DTTB & 2038.34 & 3472.63 & -410.20 & -952.57 & -63.72 & -7.80 \\
\hline
\end{tabular}

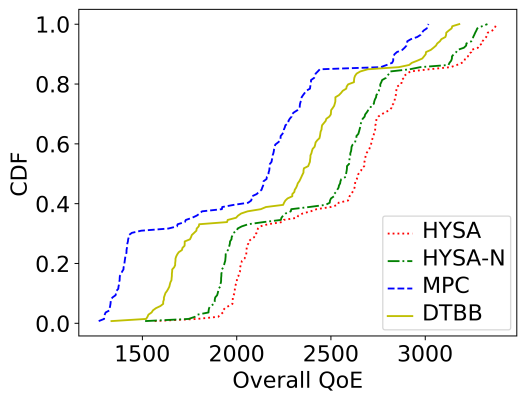

(a) Sports Video

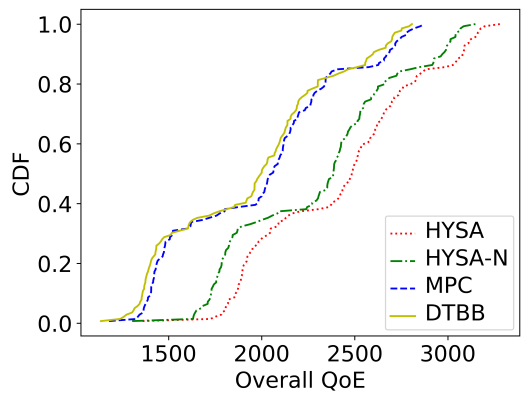

(b) Room Video

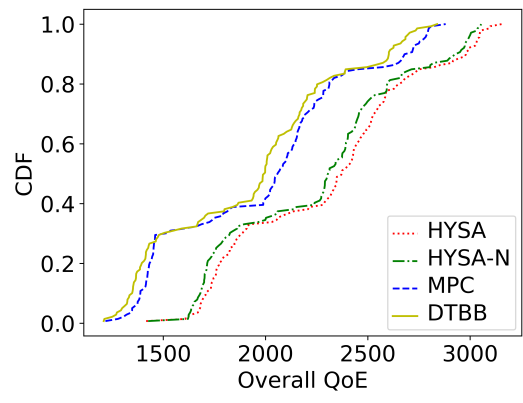

(c) Game Video

Figure 4: Overall QoE when streaming three types of videos

$$
l_{n+1}>\frac{\left(p_{q} V_{n+1, \text { qualit }_{n+1}}+p_{s}\right) d_{f}}{p_{d} \lambda}
$$

Thus, the latency threshold latency_limit ${ }_{n+1}$ that triggers frame skipping when downloading the next segment can be set as:

$$
\text { latency_limit }_{n+1}=\frac{\left(p_{q} V_{n+1, \text { qualit }_{n+1}}+p_{s}\right) d_{f}}{p_{d} \lambda}
$$

\section{EVALUATION}

In this section, we present the extensive evaluations, by which the following questions can be answered: (1) Is the segment bitrate prediction helpful? (2) How does HYSA compare to existing adaptation schemes?

To evaluate the performance of HYSA for streaming different types of videos, video traces in three scenes including room, game and sports are used for the evaluations. These videos are encoded at bitrates in $\{500,850,1200,1850\} \mathrm{kbps}$. Besides, our evaluations use 140 network traces sampled from real network scenario. The average bandwidth of these network traces covers from $0.8 \mathrm{Mbps}$ to $2.5 \mathrm{Mbps}$, while variance covers from $0.1 \mathrm{Mbps}$ to $2.0 \mathrm{Mbps}$.

First, experiments are conducted to evaluate the accuracy of KAMA-based segment bitrate prediction by calculating the prediction error $\frac{\mid \text { PredictedBitrate-ActualBitrate } \mid}{\text { ActualBitrate }}$. The results show that it can reduce the prediction error to 0.22 , against the prediction error of 0.258 when using the segment's coding bitrate for prediction directly.

Then, we compare HYSA to the following adaptation schemes using the simulator mentioned previously: (1) HYSA-N: our baseline scheme where segment bitrate prediction is not included. (2) MPC [8]: uses buffer occupancy and throughput predictions to select the bitrate which maximizes a given QoE metric over a horizon of five future chunks. (3) DTTB[7]: selects video bitrate based on a dynamic buffer threshold adapted according to the estimated throughput. Table 1 provides the average value of overall QoE and other QoE metrics that each scheme achieves on the entire network traces and video traces. Figure 4 gives the Cumulative Distribution Function (CDF) of each network trace when streaming three types of videos. From these evaluations, we can easily draw two conclusions. Firstly, the average overall QoE is improved with the KAMA-based segment bitrate predictions rather than the coding bitrates, which demonstrates that the KAMA-based segment bitrate prediction contributes to making better decisions. Secondly, the proposed hybrid control scheme outperforms other schemes with respect to overall QoE when streaming video in different scenes under various networks, because it tends to choose higher quality to improve bandwidth utilization, as illustrated by Table 1 .

\section{CONCLUSION}

In this paper, we have presented HYSA - an effective hybrid control scheme consisting of heuristic playback rate control, latencyconstrained bitrate control and QoE-oriented adaptive frame dropping. Our algorithm adopts KaufmanâĂŹs Adaptive Moving Average to predict the segment bitrates, with which we could make the bitrate decisions more accurately. Extensive simulation results have demonstrated that the segment bitrate prediction is advantageous in making better decisions, and HYSA can achieve higher overall QoE than the prior state-of-the-arts.

\section{ACKNOWLEDGMENTS}

This work was supported in part by the National Natural Science Foundation of China (Grant No. 61971382) 


\section{REFERENCES}

[1] Zahaib Akhtar, Yun Seong Nam, Ramesh Govindan, Sanjay Rao, Jessica Chen Ethan Katz-Bassett, Bruno Ribeiro, Jibin Zhan, and Hui Zhang. 2018. Oboe: autotuning video ABR algorithms to network conditions. In Proceedings of the 2018 Conference of the ACM Special Interest Group on Data Communication. ACM, 44-58.

[2] Mingfu Li, Chien-Lin Yeh, and Shao-Yu Lu. 2018. Real-time OoE monitoring system for video streaming services with adaptive media playout. International fournal of Digital Multimedia Broadcasting 2018 (2018).

[3] Hongzi Mao, Ravi Netravali, and Mohammad Alizadeh. 2017. Neural adaptive video streaming with pensieve. In Proceedings of the Conference of the ACM Special Interest Group on Data Communication. ACM, 197-210.

[4] Konstantin Miller, Abdel-Karim Al-Tamimi, and Adam Wolisz. 2017. QoE-based low-delay live streaming using throughput predictions. ACM Transactions on
Multimedia Computing, Communications, and Applications (TOMM) 13, 1 (2017), 4.

[5] Yueshi Shen, Ivan Marcin, Josh Tabak, Abhinav Kapoor, Jorge Arturo Villatoro, and Jeff Li. 2018. Buffer reduction using frame dropping. US Patent App. 10/015,224.

[6] Kevin Spiteri, Rahul Urgaonkar, and Ramesh K Sitaraman. 2016. BOLA: Nearoptimal bitrate adaptation for online videos. In IEEE INFOCOM 2016-The 35th Annual IEEE International Conference on Computer Communications. IEEE, 1-9.

[7] Lan Xie, Chao Zhou, Xinggong Zhang, and Zongming Guo. 2017. Dynamic threshold based rate adaptation for HTTP live streaming. In 2017 IEEE International Symposium on Circuits and Systems (ISCAS). IEEE, 1-4.

[8] Xiaoqi Yin, Abhishek Jindal, Vyas Sekar, and Bruno Sinopoli. 2015. A controltheoretic approach for dynamic adaptive video streaming over HTTP. In ACM SIGCOMM Computer Communication Review, Vol. 45. ACM, 325-338. 